\title{
Designing a clinical audit tool to measure processes of pregnancy care
}

\author{
This article was published in the following Dove Press journal: \\ Nursing: Research and Reviews \\ | December 201 | \\ Number of times this article has been viewed
}

\author{
Suzanne V Sinni' \\ Wendy M Cross ${ }^{2}$ \\ Euan MWallace ${ }^{1,3}$ \\ 'Department of Obstetrics and \\ Gynaecology, Monash University \\ and Southern Health, Monash Medical \\ Centre, Clayton, Victoria, ${ }^{2}$ School \\ of Nursing and Midwifery, Monash \\ University, Clayton, Victoria, ${ }^{3}$ The \\ Ritchie Centre, Monash Institute of \\ Medical Research, Monash University, \\ Clayton, Victoria, Australia
}

Correspondence: SV Sinni

Department of Obstetrics and Gynaecology, Monash University and Southern Health, Monash Medical

Centre, 246 Clayton Road,

Clayton, Victoria 3168, Australia

Email suzanne.sinni@monash.edu
Abstract: This paper reports the development of a clinical audit tool as part of a larger project to evaluate a new maternity service, underpinned by a patient safety framework.

Aim: The aim of this work is to describe the development of a clinical audit tool that measures the process of pregnancy care, and its application.

Background: There are many reports about outcomes of healthcare provision, however there are limited studies examining the process of care. There is also limited evidence linking clinical audit with improvements in care delivery. Pregnancy care was chosen because there are well defined and agreed clinical standards against which to measure the delivery of pregnancy care. A clinical audit using these standards addresses both gaps in the literature.

Methods: Standard methodological processes were used to develop the audit tool. Literature informed the processes. Data were collected in 2009-2010 using the tool described in the paper. Reliability testing was completed in September 2011.

Results: An audit tool to measure pregnancy care was developed and applied to 354 health records to enable analysis of adherence to organizational expectations of care. Reliability testing of the tool achieved an overall kappa of 0.896 .

Conclusion: Developing an audit tool based on processes described in the literature is labor intensive and resource dependent, however it results in a robust, reliable, valid tool that can be used in diverse maternity services. Stakeholder participation from the outset ensures ongoing engagement for the duration of a clinically based project spanning several years.

Keywords: clinical audit, mixed methods, maternity, patient safety

\section{Introduction}

This paper describes the development of a clinical audit tool that was a component of a larger mixed-method research design to evaluate a new maternity service. Patient safety concepts underpinned the research, which examined the processes of care during uncomplicated pregnancy. Thus, the research attempted to measure pregnancy care using clinical audit, filling a gap in the literature.

This approach was in contrast to examining outcome data, which do not provide information about the process of care afforded an individual, whether as an inpatient or in ambulatory care settings. While outcome data are certainly important, identifying deficiencies in health outcomes, giving rise to numerous healthcare inquiries, ${ }^{1-3}$ and ultimately leading to valuable recommendations regarding required improvements in patient care, there remains little agreement on how to measure it. ${ }^{4,5}$ This research will fill a gap in the literature about how the process of care is measured along the continuum of pregnancy care provision. While the subject matter is maternity care, 
the steps to develop the tool described are equally applicable in all areas of healthcare. As part of a larger evaluation, the tool discussed is detailed and complex; however nurses and midwives can apply the methodology to smaller clinicallybased activities to improve patient care.

\section{Background}

The health service where the research took place provides maternity care at three general hospitals and includes a major referral center for other facilities in Victoria (Australia) and interstate. Pregnancy (antenatal) care is provided by midwives, specialist obstetricians and medical trainees. One of the three hospitals was opened in 2004, supporting 500 women with low-risk pregnancies in the first year of operation, gradually increasing to 1500 annually. Maternity services comprise one component of broader service delivery that includes general and specialty medicine and surgery, pediatrics, mental health and aged care across 40 sites. Over 12,700 staff services a local population of over 1.2 million. Annually, 180,000 patients are admitted for all types of medical, surgical, palliative and supportive care. There are over 154,000 emergency presentations and over 8,400 births within the organization.

An evaluation of the maternity service at the new hospital was included in the planning with the regulatory authority - State government Department of Health - that began two years before the hospital opened. Stakeholders involved in the planning process included staff from the health service and the regulatory authority. Members of the research team were involved in the planning process and engaged midwives, nurses, obstetricians, pediatricians and hospital administrators to develop an evaluation framework underpinned by patient safety principles. After both consideration of the relevant literature, including regulatory requirements to meet accreditation standards, and broad consultation, it was agreed to undertake a mixed-method approach. This included a clinical audit to measure the process of care throughout normal pregnancy against previously described and agreed standards of care.

Qualitative aspects of the project included focus groups and semi-structured in-depth interviews with staff. The qualitative methodology and data will be reported separately.

\section{Aims of the study}

The aim of this study was to develop a clinical audit tool to measure pregnancy (antenatal) care at a new hospital.

The aim was informed by the hypotheses of the broader study, that:
- variation in healthcare outcomes reflects variation in practice,

- not all variation in practice is reflected in outcome data,

- measuring outcomes alone provides limited information about the overall quality of healthcare.

\section{Literature review}

Literature relevant to the work included academic publications and local documents that indicate expected clinical care within the health service where the research was conducted (hereafter referred to as "the organization"). These included clinical practice guidelines, protocols, procedures, health record documents, and charts for recording health care. Literature previously sourced to establish the framework of the project was also used. ${ }^{6}$ Peer-reviewed literature published up to September 2011 was accessed via electronic data-bases CINAHL, Ovid MEDLINE and Proquest. Subject headings used to search databases included antenatal care, maternity, midwifery, obstetrics, pregnancy, prenatal care, audit in maternity care, perinatal audit, process of care, measuring quality of care, measuring clinical outcomes, clinical outcomes, measuring clinical process. The search yielded 4028 results. Titles not related to clinical audit or measurements were excluded. References that were previously critiqued and relevant to this paper were included. Articles of empirical research as well as scholarly papers, government publications and documents from the organization were included. Subject matter included clinical audit, measurement in healthcare, maternity care and patient safety. The literature provided valuable information about clinical audit but we were unable to find any that could be directly applied in the organizational context. We therefore utilized the literature to inform the development of a specific tool.

\section{Clinical audit}

Clinical audit is defined as a systematic process to review patient care against defined and agreed criteria to identify practice gaps. These practice gaps are seen as opportunities for improvement, which when implemented result in better patient care that can be measured by re-audit. It is purported to be a key quality improvement activity that facilitates critical reflection by healthcare providers. Clinical audit is described as cyclical in nature. Processes have been variously described $^{7-14}$ and summarized in Table 1.

A variety of approaches to the development of audit criteria have been suggested. Graham ${ }^{11}$ promotes criterionbased clinical audit for near misses in obstetric complications. Examples include management of primary postpartum 


\section{Table I}

Steps to develop a clinical audit:

I. Identify relevant stakeholders to oversee the audit process

2. Identify the audit topic

3. Identify aspects of best practice that should be included in the audit

4. Agree standards and criteria against which data are measured

5. Collect the data

6. Analyze the data to ascertain the degree of compliance with standards

7. Implement improvement strategies to address practice gaps identified in the data analysis

8. Re-audit to assess the impact of improvement initiatives

9. Write and disseminate audit results

10. Re-audit regularly to assess ongoing compliance

II. Stop auditing when consistent compliance is demonstrated

hemorrhage (PPH), management of obstructed labor, or pre-eclampsia leading to eclampsia, each of which have a well-established protocol to prevent complications or death. A clinical audit would be developed based on steps in those protocols that assess compliance. Patel ${ }^{12,13}$ describes how criteria can be identified which trigger the need for clinical audit to further interrogate substandard practice and identify opportunities for improvement. A trigger using the previous example of PPH might be that all PPHs greater than $2000 \mathrm{~mL}$ must be audited to assess compliance with the local protocol. To avoid the perception of audits as irrelevant checklists, Grainger ${ }^{14}$ recommends specific approaches to increase the acceptance of audits as learning tools to improve clinical care. Evidence should inform the development of standards against which care is measured, highlighting the need for specificity of criteria to improve data reliability. All agree the importance of relevant and appropriate stakeholder support to develop and implement audit processes is of particular relevance where evidence is not specific about locally adapted processes.

There are certainly many challenges for clinicians to develop relevant, low-maintenance data collection tools in the complex environments in which they work. ${ }^{12-15}$ Health records are often incomplete or illegible - itself an auditable behavior. Primarily for fiscal reasons, scheduling of work hours (whether nursing, midwifery, medical or allied health) are traditionally based on clinical care delivery rather than providing time for clinical personnel to participate in quality-improvement activities. Such barriers are well described and reflect reality for many clinicians. However a number of approaches to overcoming these barriers have been suggested.

Nonetheless, an inability to overcome such barriers may contribute to the recurrent findings of Cochrane reviews ${ }^{15,16}$ that there is no evidence to correlate improved clinical care and audit activity despite an obvious expectation that audit feedback would influence clinician behavior. The most recent review $^{15}$ included 118 studies that demonstrated rates of compliance with desired practice varied from decreases of $16 \%$ to increases of $70 \%$, depending on the degree of support and reinforcement of implementation processes. The authors reasserted findings of the first review - that clinical audit alone does not lead to improved outcomes. Further, they argue that clinical audit should not be mandated, especially where clinical staff have not had input in the development of an audit process, unless a clear process to act on results is included in the activity. Pattinson et $\mathrm{al}^{17}$ reported similar findings on clinical audit in perinatal care. The authors supported the need for critical review of patient care but argued that feedback to clinicians who provide the care, and their ability to be involved in developing improvement initiatives, is critical in any audit process.

\section{Reliability and validity of the clinical audit}

Reliability is the ability to replicate results consistently. ${ }^{7,8,18-20}$ Inter-rater reliability is a measure of consistency between investigators using the same tool, such as a health record review in a clinical audit. Engel et $\mathrm{al}^{21}$ used available theory to develop a health record review methodology framework to standardize processes and account for inter-rater reliability. The model included an investigator, a data abstractor, an abstraction tool, an abstraction manual, a data source, and data quality analysis with each component accounted for in the research design to improve data extraction. Their model was applied to 41 health records and inter-rater reliability was calculated for each variable measured. They report the previously mentioned challenges of health record review, including availability of records, incomplete or missing information, conflicting information on the same criteria, bias of the record taker, and illegibility, and they have also processes to mitigate the challenges.

Validity is the degree to which the audit/research actually measures what it sets out to do. ${ }^{7,8,18}$ Richards ${ }^{18}$ explains that the reader should be able to follow how researchers have derived their interpretation of and conclusions from their data. Further, the researchers should provide a succinct and persuasive argument for their findings, to demonstrate that their approach is reliable and their interpretation methodical.

\section{Face and content validity}

All authors emphasize the importance of stakeholder engagement to ensure ongoing success and relevance of an audit process. ${ }^{12-16}$ Focus group interviews can be useful at the start 
of a research project to engage stakeholders, assist in research design and ensure ongoing commitment to the process. ${ }^{8}$

\section{Procedures}

\section{Ethical considerations}

Approval to undertake this study was granted by the relevant Standing Committee on Ethics in Research Involving Humans on March 13, 2007. A variation was approved on October 26, 2009, valid to October 26, 2012.

\section{Focus groups}

An initial focus group with key stakeholders provided the opportunity to explore options to evaluate the new maternity service and the scope of the project. Service planning commenced prior to opening the new hospital in 2004. In this context, participants engaged with the planning processes were invited to a meeting in January 2005 to discuss an evaluation methodology. Papers summarizing how the organization and the regulatory authority arrived at the service agreement for the new maternity service were distributed before the meeting. Information about existing tools to evaluate processes used by the organization was also sent, along with an agenda. The meeting was facilitated by the Director of Nursing. Deliberations were noted by the principal researcher as they were discussed, then clarified to ensure accurate representation. These data were then synthesized by the principal researcher and analyzed by the research team. The research team was guided by patient safety literature to develop a proposed methodology. Interpretations and proposals were emailed to participants to keep them informed of progress. Three further focus groups enabled clarification and refinement of proposed methodologies. During these meetings, the principal researcher kept notes of discussions, which were then summarized and distributed to participants. Individuals made themselves available for ad hoc consultation for the duration of the project, which enabled ongoing dialog to improve project design and reinforce the importance of the project. While the focus groups were useful in developing and refining the methodology, their main contribution to the research was the qualitative component where perceptions of the safety and quality of the new maternity service were explored which will be reported in a separate paper. This approach ensured ongoing traction and engagement with the project.

\section{Identifying clinical standards for the clinical audit tool}

The intent of the tool design was to measure care provided throughout pregnancy, based on accepted clinical practice standards for women with low risk pregnancies. Standards of maternity care in Victoria are described in various publications detailing professional, clinical, and organizational standards and were accessed for the project. These included the midwifery scope of practice, ${ }^{22}$ expectations of codes of professional conduct, ${ }^{23}$ the Royal Australian and New Zealand College of Obstetrics and Gynecology (RANZCOG) College Statements, ${ }^{24}$ the Australian College of Midwives National Midwifery Guidelines for Consultation and Referral, ${ }^{2}$ and consensus guidelines for antenatal care in low risk women developed by the three centers' collaboration. ${ }^{26}$ Local clinical practice guidelines, protocols ${ }^{27}$ and the organizational context influenced the development of the tool, as did the documents used to record clinical care at the organization. Many of these documents refer to information from the National Institute for Health and Clinical Excellence, ${ }^{28}$ particularly the Antenatal Care Guideline and advice in relation to maternity care. As per many references in the literature, where there is paucity or low-level evidence available to inform clinical practice, the organization uses consensus among key clinical staff to develop relevant tools to guide clinical practice and organizational expectations. Healthcare accreditation standards ${ }^{29}$ were also referred to in designing audit criteria.

\section{The clinical audit tool}

Recommendations from the literature guided the audit tool development, some of which has been summarized. The assessment of the processes of pregnancy care was via health record audit, based on agreed assumptions after consultation with relevant clinicians. This process accounts for hospital capabilities to release staff from clinical duties, and availability of information. The main assumptions are listed in Table 2, which also indicates sources underpinning the assumptions.

To facilitate data collection, the order of questions in the audit tool reflected the order in the antenatal record ${ }^{27}$ as much as possible. Questions were based on each clinical process. Questions were answered in either the affirmative or the negative. Where multiple responses were possible, these were divided into subsections. Figure 1 demonstrates how questions and possible responses were constructed for the audit tool.

A pilot electronic health record audit tool was developed in December 2007 after eighteen months of consultation and consideration of information from the literature. An initial pilot audit of fifty health records provided the opportunity to refine audit questions. This process was repeated three 
Table 2

Assumptions
All care provided to a woman and her
family is documented in the health record
Absent/omitted documentation assumes
care was not provided
All care provided is informed by locally
developed protocol/clinical practice
guidelines/accepted standards within the
organizational context and capability.
Women are screened to ensure they meet
inclusion criteria for an appropriate
maternity model of care at the time of
booking in, identified by a series of
assessment and screening regimes
to determine suitability.
All women are assessed by a maternity
care provider* before I 2 weeks gestation

A series of tests and investigations are offered to women before 12 weeks gestation.

Data was recorded as "normal" and "abnormal", where normal represents expected outcomes, and abnormal represents a deviation from expectations

Data recorded as abnormal should be referred for follow-up care.

Where this did not occur, data was recorded as "abnormal not referred". Routine pregnancy care is that which is planned in advance and does not include emergency pregnancy care.

All care recorded in the health record (ie, routine and emergency pregnancy care) was included in the audit.

A woman with an uncomplicated pregnancy will have at least 7 routine pregnancy care visits and not more than 10
Source/reference

Australian Council on

Healthcare Standards

(ACHS), Criterion 1.1.829

ACHS, Criterion I.I.8, ${ }^{29}$

Victorian Health Records Act $^{30}$

Study hospital

procedures, ${ }^{27}$

ACHS, Criteria I.I.I,

I.I. $2^{29}$

Study hospital

procedures, ${ }^{27}$

ACM consultation and

referral guidelines, ${ }^{25}$

ACHS, Criterion I.3.I ${ }^{29}$

Study hospital

procedures, ${ }^{27}$

NICE, ${ }^{28}$

ACHS, Criterion 3.I. $3^{29}$

3 Centres Consensus

Guidelines for

Antenatal Care ${ }^{26}$

Research team

Study hospital

procedures, ${ }^{27}$

3 Centres Consensus

Guidelines for

Antenatal Care, ${ }^{26}$

$\mathrm{NICE}^{28}$

Research team

Research team

3Centres Consensus

Guidelines for

Antenatal Care ${ }^{26}$

times before a database was built in November 2008. Results of the pilots were not analyzed because their intent was to improve specificity of the criteria against which care was measured. However, opportunities for improvement were identified and highlighted to care providers at regular clinical meetings. This was possible because the research team were also members of the clinical team. Examples of omissions that were fed back at clinical meetings included the need to use first trimester ultrasound scans to estimate a woman's due date, the importance of offering all women

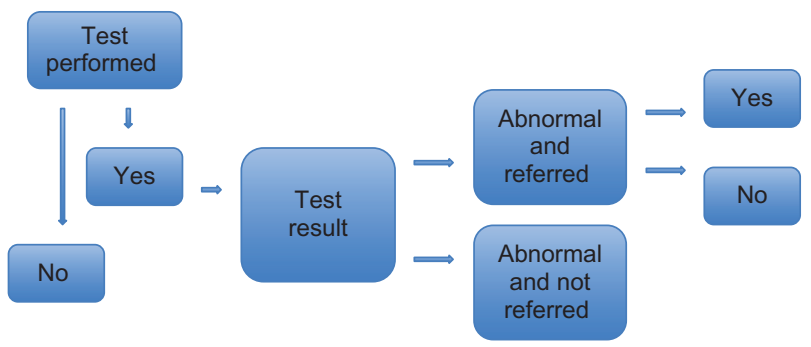

Figure I Additional responses were developed to increase specificity for individual criteria, for example assessments of women who smoke, or of women who are Rhesus Negative, to include all possible variations of follow-up.

antenatal screening for HIV and documenting when this is refused, and the importance of assessing and documenting a woman's weight and height to determine her body-mass index at the first antenatal visit. Detailed analyses will be reported separately. A copy of the audit tool is attached as Appendix 1.

\section{The clinical audit process}

Two research assistants were recruited to undertake the audit. The principal researcher met with them individually to explain the audit process, introduce the tool and detail expectations about completing the audit. Relevant authorizations for data access were arranged. The first (RA1) joined the research team in April 2009. As a resident medical officer in obstetrics and gynecology with experience in a maternity service at another organization, RA1 was invited to sample the audit tool and make further refinements, which were accepted by the research team as final in May 2009. The second (RA2), a midwife, joined the team in November 2009. RA1 audited health records when their work schedule permitted, whereas RA2 was exclusively engaged to audit health records over a 2-week period.

The first cohort of cases audited by RA1 included 155 births completed immediately prior to 31 January 2009 at the new service. 189 cases were also audited from the tertiary referral service. These data provided an opportunity for comparison across campuses as a separate project. The second cohort of cases was audited by RA 2 from 200 births completed immediately prior to 30 November 2009 at the new service. During 2009, 1000 women birthed at the new service, meaning a sample size of $36 \%$ were audited. RA1 and RA2 made file notes on ambiguous interpretations or sought clarification from the principal researcher leading to the development of a data dictionary and audit protocol.

Data were combined from both cohorts of women who birthed at the new service because it was thought that care processes would not have changed over this short time 
(two months). Cases were selected by random sampling of women who birthed in the twelve months up to the dates agreed for the two cohorts. Incomplete data were removed by the principal researcher resulting in a total of 354 audited cases.

\section{Inter-rater reliability}

The principal researcher assessed inter-rater reliability by re-auditing $25 \%$ of the total case records. In May 2011, 40 cases, 20 from each of the cohorts audited by RA1 and RA2, were re-audited. While saturation was achieved, a further 50 cases ( 20 from RA1 and 30 from RA2) were re-audited in September 2011 to ensure any discrepancies were identified. An overall kappa score of 0.88 was achieved. Kappa scores for individual criteria ranged from 0.5-1.0, with $80 \%$ individual scores $>0.8$. The majority of the criteria with kappa $<0.8$ were derived from subjective interpretations requiring RAs 1 and 2 to identify the different number of antenatal care providers one woman encounters based on different styles of handwriting because signatures were illegible. Excluding these data achieved an average kappa of 0.91. Audit results were aggregated and analyzed by the principal researcher, seeking input and advice from the research team. Results will be reported in a future publication.

\section{Limitations of the clinical audit}

Challenges encountered by researchers were consistent with those documented in the literature. Interrogation of each health record took approximately 20 minutes to complete if data were recorded where the health record review commenced. The research team agreed data would be obtained from the antenatal (pregnancy care) record. The antenatal record is a document that is intended to provide a comprehensive summary of tests, investigations and assessments of a woman during her pregnancy, a copy of which a woman carries during her pregnancy. ${ }^{28,31}$ If data were absent, it was agreed that the reviewer would further interrogate the health record to find the information. It was agreed that any reference to a test being performed and/or its result, whether in the form of a pathology report or embedded in a letter of correspondence from a referring care provider, would be accepted as evidence that a test had been performed. This information was not always easy to find and may have led to decisions that tests were omitted when they may have been performed. It also increased the time to review a health record by up to fifteen minutes.

Specificity of the criteria was systematically worked through by the research team. Key data such as gestation at first visit was not obvious from the antenatal record and a formula was developed to calculate the gestation based on the estimated due date and the date of the first visit. Assessment of a woman's social history is an important part of antenatal screening in order that appropriate referrals can be made early when indicated. ${ }^{27,28,31}$ The research team agreed that this assessment was inadequately reflected in the organizational documentation tool. The researchers agreed to collect basic information, but did not to use these data to analyze gaps in the quality of care.

Maternity care providers are expected to assess whether a woman smokes and encourage smoking cessation intervention where indicated, which should be reinforced during the pregnancy. ${ }^{26-28,31}$ Smoking during pregnancy is recorded in many different ways in the health record at the organization. The antenatal record requires a tick $(\sqrt{ })$ to indicate that a woman has been asked about smoking. When a woman indicates that she is a smoker, some maternity care providers note how much the woman smokes, if she is inclined to quit or reduce smoking, if she lives with anyone who smokes, if she will be encouraged to reduce or quit by those with whom she lives. Where such discussions occur, they may not always be noted in the health record. The research team adjusted the smoking criteria and possible responses to best reflect how smoking was assessed and overseen during pregnancy.

Criteria were handled in this way, with some being more complex than others to finalize. Criteria were then clustered into the following groups: demographics, first antenatal assessment, antenatal screening, pregnancy care, a summary of the visits schedule and compliance with documentation requirements.

\section{Discussion}

This paper describes the process of development of an audit tool to measure antenatal care at a hospital in Melbourne, Australia. The literature review provided frameworks to underpin study design and application. The literature emphasized the importance of research that measures the process of care. There are abundant publications about healthcare outcomes, complete with recommendations for improvements. However, there are no corresponding publications demonstrating whether such recommendations were acted upon with resultant improvements, barriers, or failures. Similarly, there is contention in the literature about the perceived inherent value of clinical audit in the absence of consistent evidence that audit necessarily leads to improvement. 
The approach taken by the research team was as described in the literature, working closely with key midwives and obstetricians involved in the delivery of maternity care. The research team were also members of the maternity care staff. While this was important in building credibility and engagement in the process, this approach took 2 years to build the tool. The health record audit took the equivalent of 2 months' full-time work hours to complete, which lasted over 8 calendar months. As described in the literature, this facilitated ongoing engagement and enabled minor improvements in assessment skills as midwives and obstetricians involved in the process had a heightened awareness of omissions that were detected in the audit, and shared their findings during informal communication with their colleagues. This was reinforced as the principal researcher provided regular updates about the project. Detailed data analysis and subsequent work will be reported in future publications.

\section{Conclusion}

Developing audit tools to measure the process of care is a laborious task. The benefit of this approach is building a process that is relevant and well supported. Contributions from the stakeholder group during the development of face and content validity ensured that the audit tool was comprehensive, usable and captured the key clinical data. Barriers widely reported in the literature were encountered by the research team, however organizational support and commitment of the research team ensured ongoing traction of the project. The comprehensive tool captures information about maternity services that would otherwise be hidden in patient files. A focus on patient safety with key recommendations for midwives and obstetric nurses can be developed on the basis of the amalgamated audit data, leading to improved patient outcomes.

\section{Disclosure}

The authors report no conflicts of interest in this work.

\section{References}

1. Bristol Royal Infirmary Inquiry, 2001. Available from: http://www. bristol-inquiry.org.uk/final_report/rpt_print.htm. Accessed November 22, 2011.

2. Confidential Enquiry into Maternal and Child Health. Dec 2007. Saving Mothers' Lives: reviewing maternal deaths to make motherhood safer 2003-2005. The Seventh Report of the Confidential Enquiries into Maternal Deaths in the United Kingdom. Available from: http://www.mdeireland. com/pub/SML07_Report.pdf. Accessed November 22, 2011.

3. Douglas Inquiry: Implementation of the Douglas Inquiry Recommendations Review, King Edward Memorial Hospital. Available from: http://www.kemh.health.wa.gov.au/general/KEMH_Inquiry/index.htm. Accessed November 22, 2011.
4. Pronovost P, Nolan T, Zeger S, Miller M, Rubin H. How can clinicians measure safety and quality in acute care? Lancet. 2004;363(9414): 1061-1067.

5. Australian Commission on Safety and Quality in Health Care Measurement for improvement toolkit. 2006. Available from: http:// www.safetyandquality.gov.au/internet/safety/publishing.nsf/Content/ 703C98BF37524DFDCA25729600128BD2/\$File/Toolkit_PartA.pdf. Accessed November 22, 2011.

6. Sinni SV, Wallace EM, Cross WM. Patient safety: A literature review to inform an evaluation of a maternity service. Midwifery. 2010. [Epub ahead of print.] doi:10.1016/j.midw.2010.11.001.

7. Creswell J. Research Design. Qualitative, Quantitative and Mixed Methods Approaches. 3rd ed. London: Sage; 2009.

8. Schneider Z, Whitehead D, Elliot D, editors. Nursing and Midwifery Research. Methods and Appraisal for Evidence Based Practice. 3rd ed. Sydney: Mosby; 2007.

9. Ashmore S, Ruthven T. Clinical Audit: a guide. Nurs Manag (Harrow). 2008;15(1):18-22.

10. Benjamin A. Audit: how to do it in practice. BMJ. 2008;336(7552): 1241-1245.

11. Graham WJ. Criterion-based clinical audit in obstetrics, bridging the quality gap? Best Pract Res Clin Obstet Gynaecol. 2009;23(3):375-388.

12. Patel S. Identifying best practice principles of audit in health care. Nurs Stand. 2010;24(32):40-48.

13. Patel S. Achieving quality assurance through clinical audit. Nurs Manag (Harrow). 2010;17(3):28-35.

14. Grainger A. Clinical audit: shining a light on good practice. Nurs Manag (Harrow). 2010;17(4):30-33.

15. Jamtvedt G, Young J, Kristoffersen D, O’Brien M, Oxman A. Audit and feedback: effects on professional practice and health care outcomes. Cochrane Database Syst Rev. 2006;2:CD000259.

16. Thomson O'Brien MA, Oxman AD, Davis DA, Haynes RB, Freemantle N, Harvey EL. Audit and feedback versus alternative strategies: effects on professional practice and health care outcomes. Cochrane Database Syst Rev. 2000;2:CD000260.

17. Pattinson RC, Say L, Makin JD, Bastos MH. Critical incident audit and feedback to improve perinatal and maternal mortality and morbidity. Cochrane Database Syst Rev. 2005;4:CD002961.

18. Richards L. Handling Qualitative Data. A Practical Guide. 2nd ed. Thousand Oaks CA: Sage Publications; 2009.

19. Teddlie C, Tashakkori A, editors. Foundations of Mixed Methods Research. Integrating Quantitative and Qualitative Approaches in the Social and Behavioral Sciences. Thousand Oaks CA: Sage Publications; 2009.

20. Viswanathan M. Measurement Error and Research Design. Thousand Oaks CA: Sage Publications; 2005.

21. Engel L, Henderson C, Fergenbaum J, Colantonio A. Medical Record Review Conduction Model for Improving Interrater Reliability of Abstracting Medical-Related Information. Evaluation \& the Health Professions. 2009;32:281-291. Available from: http://ehp.sagepub.com/ content/32/3/281. Accessed November 22, 2011.

22. Australian Nursing and Midwifery Council. National Competency Standards for Midwives (2008). Available from: http://www.nursingmidwiferyboard.gov.au/Codes-Guidelines-Statements/Codes-Guidelines. aspx. Accessed November 22, 2011.

23. Australian Health Practitioners Regulation Agency. Available from: http://www.ahpra.gov.au/.

24. Royal Australian and New Zealand College of Obstetrics and Gynaecology. College Statements. Clinical Obstetrics. Available from: http://www.ranzcog.edu.au/the-ranzcog/policies-and-guidelines/ college-statements.html. Accessed November 22, 2011.

25. Australian College of Midwives. National Midwifery Guidelines for Consultation and Referral. 2nd ed. Available from: http://www.midwives. org.au/scripts/cgiip.exe/WService=MIDW/ccms.r?PageId=10037. Accessed November 22, 2011.

26. Three Centres Collaboration. Consensus Guidelines. Available from: http://3centres.com.au/. Accessed November 22, 2011. 
27. Southern Health Maternity Clinical Practice Guidelines. Available from: http://www.southernhealth.org.au/page/GPs/Maternity/Maternity_ guidelines. http://www.southernhealth.org.au/page/Health_Professiona 1s/Maternity/Models_of_maternity_care/. Accessed November 22, 2011.

28. National Institute for Health and Clinical Excellence. Antenatal Care Guideline, CG62. Available from: http://www.nice.org.uk/cg62. Accessed November 22, 2011.

29. The Australian Council on Healthcare Standards (ACHS) Evaluation and Quality Improvement Program 5 (EQuIP5) Guide. 2010. Available from: http://www.achs.org.au/equip5. Accessed November 22, 2011.
30. Victorian Health Records Act 2001. Available from: http://www.health. vic.gov.au/healthrecords/0102em.pdf. Accessed November 22, 2011.

31. Primary Maternity Services In Australia. A Framework for Implementation. Australian Health Minister's Advisory Council. 2008. Available from: http://www.ahmac.gov.au/cms_documents/ Primary\%20Maternity\%20Services\%20in\%20Australia.pdf. Accessed November 22, 2011. 


\section{Appendix I \\ Pregnancy care audit}

This is a representation of a database used to undertake a pregnancy care audit in a hospital in Melbourne, Victoria, Australia. Possible responses include set data labels and free text to insert dates. In the representation below, blank boxes denote fields where free text is required.

The order of audit questions mirror organizational charts used by clinicians in their assessment of women during pregnancy care. This is not an exhaustive list. The questions were agreed by stakeholders and refined after pilots. Pregnancy care at this organization is provided by midwives, staff and consultant obstetricians, medical trainees, and General Practitioners.

The audit is divided into (1) demographics, (2) first visit, (3) antenatal screening, (4) pregnancy care, (5) visits summary and (6) documentation.

"Normal" values are well defined. There is an expectation that abnormal results will be referred for further investigation.

In auditing pregnancy care, where any assessment is omitted (or not documented) at one or more visits, the result is recorded as "no" or "not available".

1. Demographics

\begin{tabular}{l}
\hline Identifier \\
Date of birth \\
Age at first visit \\
Country of birth \\
Interpreter required \\
\hline
\end{tabular}

\section{First visit}

A Victorian (state-wide) guideline recommends that estimated due date (EDD) should be taken from the estimation provided by an ultrasound scan (USS) before fourteen weeks gestation.

\section{Date of first visit \\ EDD recorded \\ EDD calculated by}

EDD

Date of first visit Gestation at first visit Medical history taken Medical history result

\section{Yes/no}

- last menstrual period (LMP)

- Ist trimester USS and LMP

- 2nd trimester USS

- invitro fertilisation

- unknown
- Ist trimester USS

- 2nd trimester USS and LMP

Body-mass index (BMI) calculated $\mathrm{BMI}$ result

Model of care

Appropriate for model

Weight

Height

BMI

Alcohol use asked

Drug use asked

Drug use result

Physical examination undertaken

Physical examination result

Pap smear history taken

Pap smear result

3. Antenatal screening

Organizational expectations of antenatal screening is informed by available evidence or consensus and recommends that all women should be offered a series of tests and investigations.

\begin{tabular}{ll}
\hline Rhesus (Rh) negative & Yes/no \\
\hline Anti-D given & - Not applicable \\
- Anti-D administered at 28 and \\
& 34 weeks gestation (/40) \\
- & Rh antibodies (abs) at 28/40, anti-D \\
& administered at 28/40 and 34/40 \\
\hline
\end{tabular}


(Continued)

Full blood examination (FBE) taken

FBE result

Syphilis screening done Syphilis result

Hepatitis B screening done Hepatitis $B$ result

Rubella screening done Rubella Result

Hepatitis $C$ screening done Hepatitis C result

HIV screening done

HIV result

Mid stream urine (MSU)

test done

MSU result

Maternal serum screening

(MSS) done

MSS result

USS before 14/40 done

USS $<14 / 40$ result

Glucose challenge test (GCT) done
- Rh abs at 28/40, anti-D administered at $28 / 40$

- Rh abs at 34/40, anti-D administered at $28 / 40$ and $34 / 40$

- Anti-D at $28 / 40$

- Anti-D at 34/40

- No

Yes/no

- Normal

- Abnormal and referred

- Abnormal and not referred

- Not available

Yes/no

- Normal

- Abnormal and referred

- Abnormal and not referred

- Not available

Yes/no

- Normal

- Abnormal and referred

- Abnormal and not referred

- Not available

Yes/no

- Normal

- Abnormal and referred

- Abnormal and not referred

- Not available

Yes/no

- Normal

- Abnormal and referred

- Abnormal and not referred

- Not available

Yes/no

- Normal

- Abnormal and referred

- Abnormal and not referred

- Not available

Yes/no

- Normal

- Abnormal and referred

- Abnormal and not referred

- Not available

Yes/no

- Normal

- Abnormal and referred

- Abnormal and not referred

- Not available

Yes/no

- Normal

- Abnormal and referred

- Abnormal and not referred

- Not available

Yes/no
(Continued)

\begin{tabular}{ll}
\hline GCT result & - Normal \\
& - Abnormal and referred \\
& - Abnormal and not referred \\
& - Not available \\
& Yes/no \\
& - Normal \\
USS 18-20/40 done & - Abnormal and referred \\
USS 18-20/40 result & - Abnormal and not referred \\
& - Not available \\
\hline
\end{tabular}

4. Pregnancy care

Organizational expectations of pregnancy care include assessments at every antenatal visit.

\begin{tabular}{|c|c|}
\hline Blood Pressure (BP) & Yes/no \\
\hline \multicolumn{2}{|l|}{ assessed at every visit } \\
\hline \multirow[t]{5}{*}{$\mathrm{BP}$ result } & - Normal \\
\hline & - Abnormal and referred \\
\hline & - Abnormal and not \\
\hline & referred \\
\hline & - Not available \\
\hline Symphysis-fundal height (SFH) & Yes/no \\
\hline \multicolumn{2}{|l|}{ assessed at every visit after $20 / 40$} \\
\hline \multirow[t]{5}{*}{ SFH result } & - Normal \\
\hline & - Abnormal and referred \\
\hline & - Abnormal and not \\
\hline & referred \\
\hline & - Not available \\
\hline Abdominal palpation (abdo palp) & Yes/no \\
\hline \multicolumn{2}{|l|}{ performed every visit after 30 weeks } \\
\hline \multirow[t]{5}{*}{ Abdo palp result } & - Normal \\
\hline & - Abnormal and referred \\
\hline & - Abnormal and not \\
\hline & referred \\
\hline & - Not available \\
\hline Fetal movement (FM) assessment & Yes/no \\
\hline every visit & \\
\hline \multirow[t]{5}{*}{ FM result } & - Normal \\
\hline & - Abnormal and referred \\
\hline & - Abnormal and not \\
\hline & referred \\
\hline & - Not available \\
\hline Vitamin $\mathrm{K}$ discussed & Yes/no \\
\hline Hepatitis B vaccination discussed & Yes/no \\
\hline Smoker follow-up & Auto-populated from first \\
\hline & visit screen \\
\hline Number of antenatal carers & \\
\hline
\end{tabular}

\section{Visit summary}

The hospital where the audit was undertaken provides low risk pregnancy care. Organizational expectations of the number of antenatal visits offered to women is informed by relevant literature. Women with low risk pregnancies should be offered seven to ten pregnancy visits. The possible number of visits was based on actual numbers 
recorded on the health records audited. Any contact that was documented in the health record was identified as a separate visit. The first antenatal visit should be before twelve weeks gestation.

\section{Date of 2nd visit \\ Date of 3rd visit \\ Date of 4th visit \\ Date of 5th visit \\ Date of 6th visit \\ Date of 7 th visit \\ Date of 8th visit \\ Date of 9th visit \\ Date of I0th visit \\ Date of I I th visit \\ Date of I2th visit \\ Date of I 3 th visit \\ Date of 14th visit \\ Date of I5th visit \\ Date of 16 th visit \\ Date of 17th visit \\ Date of I8th visit \\ Date of 19th visit \\ Date of 20th visit \\ Date of 2 I st visit \\ Date of 22nd visit \\ Date of 23rd visit \\ Date of 24th visit}

Number of antenatal visits

Number of antenatal visits before $18 / 40$

Number of antenatal visits between $18-28 / 40$

Number of antenatal visits after $28 / 40$
Gestation at 2nd visit Gestation at 3rd visit Gestation at 4th visit Gestation at 5th visit Gestation at 6th visit Gestation at 7th visit Gestation at 8th visit Gestation at 9th visit Gestation at 10th visit Gestation at I Ith visit Gestation at 12th visit Gestation at 13th visit Gestation at 14th visit Gestation at 15th visit Gestation at 16th visit Gestation at 18th visit Gestation at 19th visit Gestation at 20th visit Gestation at 2 I st visit Gestation at 22nd visit Gestation at 23rd visit Gestation at 24th visit Gestation at 17 th visit

\section{Documentation}

\begin{tabular}{lc}
\hline Every page has a bradma (unit record number) & Yes/no \\
Every entry is dated & Yes/no \\
Every entry is signed & Yes/no \\
Every signature is accompanied by a legible signature & Yes/no \\
Every entry is legible & Yes/no \\
\hline
\end{tabular}

Nursing: Research and Reviews

\section{Publish your work in this journal}

Nursing: Research and Reviews is an international, peer-reviewed, open access journal publishing original research, reports, reviews and commentaries on all aspects of nursing and patient care. These include patient education and counselling, ethics, management and organizational issues, diagnostics and prescribing, economics and

\section{Dovepress}

resource management, health outcomes, and improving patient safety in all settings. The manuscript management system is completely online and includes a very quick and fair peer-review system. Visit http://www.dovepress.com/testimonials.php to read real quotes from published authors. 